\title{
Making Sense of the Relationship Between Adaptive Thinking and Heuristics in Evolutionary Psychology
}

\author{
Shunkichi Matsumoto ${ }^{1}$ (D)
}

Received: 27 February 2020 / Accepted: 24 October 2020 / Published online: 9 February 2021

(c) The Author(s) 2021

\begin{abstract}
In recent years, quite a few evolutionary psychologists have come to embrace a heuristic interpretation of the discipline. They claim that, no matter how methodologically incomplete, adaptive thinking works fine as a good heuristic that effectively reduces the hypothesis space by generating novel and promising hypotheses that can eventually be empirically tested. The purpose of this article is to elucidate the use of heuristics in evolutionary psychology, thereby clarifying the role adaptive thinking has to play. To that end, two typical heuristic interpretations-Machery's "bootstrap strategy" and Goldfinch's heuristically streamlined evolutionary psychology—are examined, focusing on the relationship between adaptive thinking and heuristics. The article draws two primary conclusions. The first is that the reliability of the heuristic hypothesis generation procedure (in the context of discovery) should count no less than the conclusiveness of the final testing procedure (in the context of justification) in establishing scientific facts; nature does not always get the last word. Philosophy also counts. The second is that adaptive thinking constitutes a core heuristic in evolutionary psychology that provides the discipline with its raison d'être, but this is only possible when adaptive thinking is substantiated with sufficient historical underpinnings.
\end{abstract}

Keywords Adaptive thinking · Bootstrap strategy · Contexts of discovery and justification · Division of labor · Evolutionary psychology $\cdot$ Heuristics

\section{Introduction}

The controversy revolving around evolutionary psychology does not seem to be subsiding; however, the focus of the debate has been gradually shifting. Before, the trend used to be that the debate primarily revolved around the objections raised by critics from a methodological point of view. Some problematized the stability of the Pleistocene environment as the human Environment of Evolutionary Adaptedness (EEA) that is necessary for natural selection to work out robust solutions over an evolutionary time scale (Sterelny 1995; Sterelny and Griffiths 1999; Buller 2005; Richerson and Boyd 2005).

Some others cast a question about the grain with which the ancient adaptive problems should be identified: does fear in general constitute a single adaptive problem, or should the fear of predators and that of heights be considered as

Shunkichi Matsumoto

mshunkichi@gmail.com

1 Center for Liberal Arts, Tokai University, Kanagawa, Japan separate problems to be subsumed under a related domain (Sterelny and Griffiths 1999; Buller 2005)?

Still others doubted the feasibility or logical consistency of adaptive thinking. For the purpose of identifying ancient adaptive problems with sufficient precision to be able to pick out only relevant aspects of the environment while screening out unnecessary information, we need to know quite a good deal about the trait in advance (Griffiths 1996; Buller 2005; Laland and Brown 2011).

In recent years, however, quite a few evolutionary psychologists or their defenders have come to emphasize evolutionary psychology as a scientific discipline based on heuristic predictions and eventual confirmation (Gigerenzer and Selten 2001; Andrews et al. 2002; Goldfinch 2015; Hagen 2016; Machery forthcoming). According to them, no matter how methodologically incomplete, adaptive thinking (a core methodology of evolutionary psychology to be clarified later) works fine as a good heuristic in effectively reducing the hypothesis space. The methodological objections raised by critics we just synopsized above do not doom evolutionary psychology, because they all concern the context of discovery, not the context of justification: if the hypotheses 
discovered ought to have been justified in terms of methodological consistency in advance of their final testing, those objections would surely be crucial. However, if the truthfulness of the hypotheses is to be entirely determined by final testing, it will not make any serious difference which methodology is employed in the process of discovering hypotheses, or where they come from. After all, it is not philosophy (methodology) but nature that gets the last word (Symons 1992).

For example, Edouard Machery advocates such a heuristic interpretation. According to him, what he calls "the forwardlooking heuristic" (adaptive thinking, in our terms) assumes a central place in evolutionary psychology reasoning. Yet, at the same time, he remarks that although it is useful for discovering our psychological traits, it need not be necessary. Sometimes it is supplemented by a backward-looking reasoning, and at other times its speculative character needs to be constrained by some other non-evolutionary sources of information. Since the forward-looking heuristic is just a heuristic, it need not stand on its own as a complete and self-contained hypothesis generator (Machery forthcoming).

Andrew Goldfinch brings such a heuristic aspect to the fore and argues that it is this aspect that evolutionary psychology as a scientific practice conducted by today's most pragmatic researchers on a daily basis ought to be identified with. According to his diagnosis, the reason why evolutionary psychology at its early stage provoked such fierce antipathy from critics is because its leading pioneers such as Leda Cosmides, John Tooby, David Buss, and others ventured to sell "a package of strong views" that presented it as a "game-changer," a "scientific revolution" in psychology, the unifying principle in behavioral and social sciences, or even having a bearing on public policy making. Instead, Goldfinch insists that evolutionary psychology be "streamlined" by letting go of these sorts of excessive promises unlikely ever to be fulfilled, in order to circumvent irrelevant criticisms against it. Evolutionary psychology should rather be taken as a hypothesis-driven empirical science, the daily practice of which consists in a kind of adaptationist version of the hypothetico-deductive method; that is, focusing on adaptive problems, hypothesizing dedicated solutions to the problems, and then subjecting these hypotheses to testing (Goldfinch 2015, p. 132).

However, as we will argue later, heuristics come with their own problems. Using heuristics to find solutions to given problems means committing ourselves to more or less reductive explanations to make them tractable by reducing the complexity of the system concerned. But this, in turn, makes it prone to oversimplified conceptions of its components, contexts, environments, and their interactions, likely resulting in "reductionist biases" (Wimsatt 2007).

This is especially true of evolutionary psychology where, as we will see later, adaptive thinking is feasible only in so far as some drastic simplifying assumptions are in place, such as those concerning the existence of nonselective forces, persistence of ancient selection pressures, effects of epistatic interactions, or existence of developmental or phylogenetic constraints.

The purpose of this article is to elucidate the use of heuristics in evolutionary psychology and thereby clarify the role adaptive thinking has to play. To that end, in the next section, the situation will be reviewed in which the pioneers of evolutionary psychology tried to advertise adaptive thinking as the proprietary methodology that enabled them with its heuristic function to enjoy a methodological advantage over that of beleaguered sociobiologists.

In the third section, I will take up one major methodological objection to evolutionary psychology as a case example- the charge of circular reasoning in identifying adaptive problems - and examine whether Machery's idea of "bootstrap strategy" as a response to it-that adaptive thinking can get away with the charge by being supplemented by reverse engineering — can address it properly.

In the following section, I will turn to Goldfinch's proposal of heuristically streamlined evolutionary psychology. There I will focus on his proposal of division of labor between evolutionary psychology as managing heuristic hypothesis generation and adjacent relevant fields as justifying them in order to see if it can circumvent the conventional charge that evolutionary psychological hypothesization wants evidential supports.

In the fifth section, I will introduce Matthew Rellihan's analysis of the type of adaptive thinking employed in evolutionary psychology (Rellihan 2012) in order to clarify the role of adaptive thinking and thereby identify one of the core (biasing) assumptions inherent in the program.

In the sixth section, I will readdress the initial issue of the possibility of construing evolutionary psychology as a heuristic project and what to make of the relationship between adaptive thinking and heuristics.

Two primary points will be drawn by the end of this article. The first is that the reliability of the heuristic hypothesis generation procedure (in the context of discovery) should count no less than the conclusiveness of the final testing procedure (in the context of justification) in establishing scientific facts; nature does not necessarily get the last word. Philosophy also counts. The second is that adaptive thinking constitutes a core heuristic in evolutionary psychology that provides the discipline with its raison d'être but that this is only possible when adaptive thinking is substantiated with sufficient historical underpinnings. 


\section{Adaptive Thinking in Evolutionary Psychology}

In this brief section, for the argument to follow, I will preliminarily delineate what adaptive thinking means in evolutionary psychology and how its pioneers appealed to it to establish their methodological advantage over other approaches.

Adaptive thinking is a type of reasoning in which, on the basis of prespecified selection pressures, structures or behaviors of the organism that must have been evolved as adaptive responses are inferred; it is a forward-looking inference from past functions (survival values) to current forms. Usually adaptive thinking is contrasted with reverse engineering, which infers backwardly from current forms to past functions. Put another way, "Reverse engineering infers the adaptive problem from the solution which was adopted. Adaptive thinking infers the solution from the adaptive problem" (Griffiths 1996, p. 514).

The pioneers of evolutionary psychology initially advertised their methodological advantage over that of sociobiologists of older generations by appealing to adaptive thinking, or what they called "evolutionary functional analysis" (Tooby and Cosmides 1992). Sociobiologists used to be accused of untestable post hoc storytelling about the historical origins they conjectured by the reverse engineering of currently observed traits. In contrast, evolutionary psychologists are supposed to be exempted from such accusations because the end-products of their forward-looking reasoning, namely, the psychological mechanisms possessed by modern humans, can directly be put to empirical testing. This way, adaptive thinking can better lend itself to the typical formula of hypothesis-driven scientific reasoning-focusing on adaptive problems, hypothesizing solutions to them, and finally confirming them empirically.

For example, Cosmides, Tooby, and Barkow (1992, p. 11) states that, "One virtue of this approach is that it is immune to the usual (but often vacuous) accusation of post hoc storytelling: The researcher has predicted in advance the properties of the mechanism."

Adaptive thinking is also supposed to have heuristic value. By figuring out the solutions that might have solved postulated adaptive problems, adaptive thinking is expected to lead to the discovery of previously unknown features. Thus, "an explanation for a fact by a theory cannot be post hoc if the fact was unknown until after it was predicted by the theory and if the reason the fact is known at all is because of the theory" (Tooby and Cosmides 1992, p. 75).

At the same time, it can serve as a kind of winnow to narrow down the vast hypothesis space by identifying "out of the millions of possible theories" those "that are more likely to be true" (Tooby and Cosmides 1998, p. 197), namely, by sorting out a handful of promising hypotheses from the rest of those unworthy of serious consideration in accordance with whether they make evolutionary sense.

\section{The Charge of Circular Reasoning in Identifying Adaptive Problems and Machery's Idea of the "Bootstrap Strategy"}

Many different types of criticism have been levelled against adaptive thinking as a central methodology of evolutionary psychology. Here, I will pick out one, for it concerns the feasibility - or internal logical consistency — of the forwardlooking inference itself, and thus I think is central among all. That is the problem related to identifying adaptations in our ancestral past (Rose and Lauder 1996; Buller 2005; Richardson 2007; Fox and Westneat 2010; Laland and Brown 2011). It may not be impossible to infer whether a given trait is an adaptation by conjecturing which traits might have been favored by natural selection in the past, provided that sufficient knowledge of evolutionary processes and ancestral environmental conditions are available (Cosmides and Tooby 1987; Tooby and Cosmides 1990). However, whether such conjectures can be meaningfully made in practice is a matter of controversy. Since researchers are rarely completely ignorant of the features of the trait in question, they may be in a position to cheat and fudge an evolutionary scenario that predicts features of the trait that are already known (Laland and Brown 2011, p. 133). If this is the case, the credibility of the confirmation process of those predictions where they are confronted with the current data-be it through experiments, questionnaires, or cross-cultural studies-will be compromised.

Against this conventional criticism, Machery argues that evolutionary psychologists can escape the charge by construing the whole reasoning procedure as a "bootstrap strategy" in which the preceding reverse-engineering and the following adaptive thinking work together in tandem. He writes,

Moreover, the forward-looking heuristic is often complemented by a bootstrap strategy. Evolutionary psychologists often use the knowledge accumulated by psychologists about the structure of known psychological traits to infer what past selective pressures might have been (backward-looking reasoning). These hypotheses about past selective pressures are then used to develop novel hypotheses about some properties of these known psychological traits or to attempt to discover new psychological traits (forward-looking reasoning). (Machery forthcoming, p. 8). 
Edward Hagen also endorses Machery's view:

Used separately, these two types of arguments each do have limitations. Used together, however, and in combination with well-tested theories from evolutionary biology, they are able to make genuine contributions to understanding human evolution. (Hagen 2016, p. 149)

The point is that the hypotheses about past selective pressures reached by backward-looking reasoning can then serve as a springboard for further conducting a forward-looking one for developing novel hypotheses about some properties of the traits in question. Forward-looking reasoning cannot stand alone, indeed. But with the auxiliary help of backwardlooking reasoning based on already known traits-on top of other circumstantial evidence available_-it can "boot up" and perform the desired function.

Herein lies the problem: is it really a virtuous circle as Machery and Hagen envisage, or might it be perhaps a vicious circle as critics suspect? (Caporael 1989; Davies 1999; Buller 2005) Even those proponents admit that both forward- and backward-looking reasonings are in themselves incomplete-a forward-looking one being beset with the problem of the incomplete identifiability of the EEA adaptive problems at the outset, and a backward-looking one being saddled with the problem of underdetermination by available evidence among the multiple competing hypotheses consistent with what we observe now. ${ }^{1}$ If so, can two in-themselves incomplete methods complement each other to form a more reliable one? Or might it not be the case that an uncertain inference method that builds on an in-itself uncertain premise will end up with something like a house of cards?

Since Machery does not give us concrete examples of how this strategy works, let us consider instead the case Hagen makes. Following the above quote, Hagen argues as follows to instantiate the bootstrap strategy:

The universal aspects of mate preferences of contemporary women provide a decent hypothesis for the mate preferences of ancestral women, for instance, .... These hypothesized ancestral female preferences are then essential components of the EEA of male-mating strategies of humans .... (Hagen 2016, p. 149)

This sounds slightly simplistic. First, how can he assert that those aspects of women currently observed are "universal"?

\footnotetext{
1 Bringing up one example of this latter problem (an example of the former problem is to be discussed shortly), the Archaeopteryx foot exhibits a design for grasping, but this observation alone is insufficient for determining whether it evolved to grasp branches (i.e., to perch), implying that Archaeopteryx was adapted for flight, or to grab prey, implying that it was a terrestrial predator (Richardson 2007; Hagen 2016).
}

He seems to neglect the variations existing among contemporary women (e.g., not all women prefer high-status men). Second, he identifies the ancestral female preferences hypothesized through backward-looking reasoning immediately with the essential components of the EEA constituting the male adaptive problems from which to start forwardlooking reasoning. However, the "hypothesized" preferences are not the actual ones, unless confirmed so.

Finally, if we reconstruct the reasoning presented in his sketchy argument, using standard evolutionary psychology doctrine to fill in the missing links, we would have the following chain of reasoning:

The universal features of modern women that prefer certain types of male behavioral patterns (industriousness, strife for high status, etc.) can be projected onto those of ancestral women using backward-looking reasoning.

$\rightarrow$ These projected female features in turn can be used to infer the sorts of selection pressures that contemporary men were forced to face in order to survive the intrasexual competition of the time.

$\rightarrow$ These ancient selection pressures, combined with what is predicted from Trivers's parental investment theory that men were placed under severer intrasexual competition (Trivers 1972), are supposed to serve as a springboard for the subsequent forward-looking reasoning to hypothesize the specialized psychological mechanisms that our male ancestors should have evolved by the end of the Pleistocene, in regard to a mating strategy.

$\rightarrow$ These evolved male mechanisms are what modern men are supposed to inherit virtually unchanged due to the lack of necessary time for evolution of complex adaptations after the end of the Pleistocene. ${ }^{2}$

$\rightarrow$ This explains why modern men are innately disposed to behave in a way that conforms to preferences of modern women observed at the outset.

\footnotetext{
2 According to Smith (2020), even this assumption of the sameness of the traits of our ancestors and those of modern humans naively postulated and shared by evolutionary psychologists (implicit in the first and fourth links in this chain) is enough to make us doubt the possibility of evolutionary psychology. For, without the explicit demonstration that the modern trait is descended from the ancestral one along the same lineage and therefore that the function that affects the fitness of the modern trait is nothing but the function that caused the ancient trait to be selected for-what she dubs "strong vertical homology" - the whole research program of evolutionary psychology would collapse. I agree that this is an aspect that has been overlooked even by critics, let alone evolutionary psychologists, that has a serious consequence. I will remain neutral on this issue for the time, however, just for the sake of my current argument.
} 
Now, whether this chain of reasoning as a composite of backward- and forward-looking reasonings proves to be a successful case of the bootstrap strategy to yield a novel prediction or collapses into an unproductive circularity seems to hinge upon whether there is any chance to subject the end products of this chain (i.e., predicted male mechanisms) to empirical confirmation that can be designed independently of the corresponding behavioral patterns supposed to supervene on those mechanisms (in terms of, say, identifying the underlying neuronal circuits responsible for those patterns). If, on the other hand, the intended confirmation was a mere reassurance of those patterns observed at the outset, then the whole detour to and from the ancestral environments would be redundant. Yet, at least up to the present point in time, the alleged confirmation conducted by evolutionary psychologists has not met this requirement.

For instance, let us take up Buss's well-received theory of jealousy (Buss et al. 1992; Buss 2000, 2008). This is a partial application of Trivers's theory of parental investment and sexual selection as a middle-range evolutionary theory to a specifically human case (Trivers 1972). ${ }^{3}$ According to it, the sex that is more heavily investing in offspring tends to be choosier in mate selection; whereas the less-investing sex tends to be more promiscuous and simultaneously forced into competitive intrasexual selection.

Now, on the one hand, human females are, as in most other mammalian and bird species, investing more than males; therefore, Trivers's theory applies to humans. However, on the other, there are some peculiarities among humans; as female ovulation is concealed, paternity uncertainty becomes a problem among males. In addition, human males are, differently from other primate relatives, considerably committed to parental investment, especially postnatally. Trivers's theory predicts that these factors can lead men to be "choosier" in their own manner, namely, more vigilant about the reproductive activities of their mates than other primate counterparts. If a man's partner has an affair with another man, it poses a serious threat to his reproductive prospect as he is not certain about the paternity of the child his partner bears, and, hence, he risks misallocating his resources on a child he did not father. In contrast, his partner's emotional attachment to another man is less serious as long as she is sexually faithful. On the other hand, for a woman (as the higher-investing sex and, therefore, in need of resources), her partner's emotional attachment to another woman poses a serious threat to her reproductive prospects, for then part of the resources she was supposed to receive will likely be allocated to another woman. In contrast, her partner having brief extramarital affairs is of lesser concern as long as he is emotionally faithful.

\footnotetext{
3 See also note 8 .
}

Buss predicts, from these considerations, that human males must have evolved an innate jealousy module that makes them more alert to their mates' sexual infidelities, whereas their female counterparts must have evolved one that makes them more alert to their mates' emotional infidelities.

Now let us turn to the hitherto attempted verification of this prediction. Buss and others have conducted it primarily counting on either self-reports on forced-choice questionnaires or the measurement of the physiological stress responses of male and female test subjects who were asked to imagine an uncomfortable scene where their partner, with whom the subject is deeply involved, is being (emotionally or sexually) unfaithful with another person. The researchers then reported that their predictions about sex-biased jealousy sensitivity were confirmed (Buss et al. 1992).

The problem I see is, however, whatever the resultwhether those predictions be positively or negatively confirmed-what is sought to be verified here is whether the relevant jealous emotions (or some associated bodily responses) are aroused in subjects, not whether they are brought about by some underlying mechanisms. Buss should indeed be credited for designing experiments to confirm, in a quantitative measure, the extent to which the types of jealous emotions entertained by the different sexes differ. Still, until it is demonstrated-or, at least, the experimental design is proposed to demonstrate - that the behavioral differences are caused by some underlying modules hardwired differently between the sexes, the alleged confirmation of the sex-biased sensitivity will remain as a mere reassurance or an accommodation of known facts, rather than a prediction of novel phenomena, albeit adding some quantitative underpinning.

This situation is typical of hypothesization and confirmation in evolutionary psychology; it is usually the case that the required mechanisms are presumed to lie at the underlying information-processing level as something responsible for the corresponding behavioral outputs, namely, they are postulated just as hypothetical placeholders for what we can currently observe. They do not have any chance to play substantial roles in the confirmation of hypotheses, at least for the time being, and thus are theoretically unnecessary. This will make the detour to and from the ancient EEA seem redundant; Machery's remedy against the charge of circularity does not seem promising.

\section{Goldfinch's Proposal of Heuristically Streamlined Evolutionary Psychology}

Goldfinch adds another twist to this issue. He admits that if given an explanatory interpretation, evolutionary psychology may well end up with a circular explanation consisting in projecting forward into the present that which was 
once obtained by projecting back into the past that which is currently observed. However, evolutionary psychology can manage to break loose from this vicious circle charge by being interpreted as a heuristic project, not as an explanatory project.

The key to this interpretation is a distinction between explanations and heuristic hypothesis generations. According to Goldfinch, evolutionary psychology should not be considered to provide final explanations of phenomena; rather, it should be regarded as just producing hypotheses to be confirmed later. The difference between the two can be put as follows: while explanations are expected not only to provide hypotheses but also to eventually justify them, heuristic projects can stop short of this justificatory procedure.

For instance, if one is to propose via adaptive thinking that trait $T$ is an adaptation for $X$, all that is required of heuristic projects is to make the following inference in the form of a conditional (here $X$ refers to some adaptive problem, $T$ some trait as a solution to $X, C$ some properties exhibited by $T$, and $P$ some observable phenomena derived from $C$ ): "If trait $T$ is an adaptation for $X$, trait $T$ should have configuration $C$, and so we should find phenomenon $P^{\prime \prime}$ (Goldfinch 2015 , p. 144). Making a further factual claim that trait $T$ is actually an adaptation for $X$ is not in the purview of a heuristic project, much less justifying it.

According to Goldfinch, it is because evolutionary psychology hypotheses have been unduly deemed as self-contained final pronouncements that unnecessary objections expressing doubt about them are raised. Instead, if they are considered to be just hypotheses waiting (and wanting) to be verified, then those objections will disappear, and other adjacent relevant disciplines will take up the baton and put them to the test.

I wonder if we can separate hypotheses from explanations in such a dichotomous manner. In my eyes, they are more or less mutually exchangeable concepts. In science, every time a new thus-far-unknown phenomenon is discovered, scientists try to explain it, no matter how tentative that explanation may be. Any and all explanations are fallible and left open to revision, thus assuming a hypothetical character. On the other hand, any hypotheses are products of the attempt to explain thus-far-unexplained phenomena and, therefore, are themselves already kinds of explanations, with their provisional character being emphasized. It is not that mere hypotheses waiting to be tested and full-blown explanations established as true are qualitatively separated. Hence, it does not seem that, by simply renaming the concept from "explanations" to "hypotheses," the situation will change so drastically that the critical backlash from skeptics will subside.

The situation will rather be that the probability of hypotheses becoming true propositions is a function of both the reliability of the procedure generating them (i.e., context of discovery) and the conclusiveness of the final testing procedure (i.e., context of justification). The more reliable the former procedure already is, the more likely to be true the hypothesis generated will be, and the less crucial role the latter procedure will have. In contrast, if the former is error-prone in some way or another, the evidential criteria for the eventual confirmation will have to be all the more demanding. Furthermore, it is often the case that the initial errors made in the context of discovery have an overarching biasing effect on practices done in the context of justification without being noticed by practitioners. We can substantiate this point by referring to Wimsatt's argument about the "reductionist problem-solving heuristics" (Wimsatt 2007; see also Tversky and Kahneman 1974). ${ }^{4}$

According to Wimsatt, using heuristics is applying a kind of reductionistic research strategy for reducing the complexity of the system to a tractable level by introducing simplifications, idealizations, or approximations. ${ }^{5}$ As such, it is prone to the same kind of errors or biases of reductionism in general. Among them, the most relevant to our current argument is that, "The errors produced by using a heuristic are not random but systematically biased" (Wimsatt 2007, p. 76). That is, even different heuristics slightly modified by different practitioners to get better fits tend to "generate errors in the same direction" $(2007$, p. 84$)$ if they share the initial biasing assumptions introduced by the originators of the research program.

Wimsatt goes on to argue that "heuristics can hide their tracks" (2007, p. 86). That is, those multiple reductionist models sharing the initial basic assumptions have a generic tendency to constrain the overall direction along which to expect the results in such a way that each model covers up each other's inadequacies; rather than producing independent results either confirming or disconfirming a hypothesis, heuristic models frequently create pseudo-robust conclusions confirming initial theoretical biases. Therefore, instead of fulfilling the expected function of significantly reducing the hypothesis space, the use of heuristics may often end up

\footnotetext{
${ }^{4}$ I am grateful to an anonymous reviewer for enabling me to elaborate my argument into the current form by drawing my attention to these points.

5 A generic conception of heuristics will be that of rules of thumb that "serve as guidelines for finding a solution to a given problem quickly and efficiently," at the expense of giving up making "exhaustive random trial and error searches," in a problem space comprising all possible configurations in a relevant domain (Schickore 2018; see also Gigerenzer and Selten 2001). One important feature resulting from this conception is that, differently from truth-preserving algorithms, heuristics make no guarantees that they will produce a solution (let alone a correct solution) to the problem. This further indicates that the use of heuristics does not always guarantee an effective reduction of hypothesis space but instead can make it even more confounding by adding to spurious hypotheses (cf. Tversky and Kahneman 1974), the point to be addressed in what follows.
} 
entrenching the underlying biases and thus compromising the falsifiability, as it were, of the research program.

In this same vein, Paul Griffiths (1996) notes a "negative heuristic effect" of adaptive thinking to draw attention not just to the ease with which an adaptive hypothesis can be invoked to accommodate existing or novel findings but, more importantly, to its tendency to rule out other equally plausible hypotheses borne out by different sets of findings once a particular hypothesis has become predominant. As an example, he raises the case for parent/offspring conflict that was first put forward by Trivers (1974) and immediately gained considerable momentum among sociobiologists (and has remained in some circles up to today). Although the idea that the parent wants to conserve its resources for future offspring, whereas the offspring wants as much as it can get now, is quite appealing, Griffiths notes, empirical evidence for parent/offspring tug-of-war (especially over weaning) is very weak. On the contrary, he cites Bateson's (1994) review of a number of studies that failed to find aggressive interactions at weaning in various species; namely, studies that report voluntary weaning on offspring's part or ones that found both parties signaling to each other in order to coordinate peaceful weaning, although these studies have largely been underappreciated. The point here is that a predominant hypothesis can suppress others by heuristically (i.e., selectively) picking out evidence that fits it most.

These points of argument have a great bearing on Goldfinch's proposal of division of labor between evolutionary psychology as engaging in just hypothesis generation and adjacent relevant fields as undertaking the task of justifying them: the task ought to be taken on by evolutionary psychologists themselves of systematically investigating heuristic biases and their adverse effects inherent in the program. If it is delegated to practitioners in other fields, they will more likely try to collect evidences either confirming or disconfirming the artifacts created by those biases rather than detect the underlying biases themselves that even evolutionary psychologists could not notice.

In order to substantiate these points, in the next section I will look at Rellihan's argument on the nature of "Adaptationism and Adaptive Thinking in Evolutionary Psychology" (Rellihan 2012) and bring out one of the core biasing assumptions initially introduced by the pioneers into the program.

\section{Rellihan's Analysis of Adaptationism in Evolutionary Psychology}

According to Rellihan, the type of adaptive thinking typical of evolutionary psychology is in fact what can be termed "strong adaptationism." This is the idea that the force of natural selection is so powerful and overwhelming to any obstacles that, once given perennial selection pressures, the destination of adaptive evolution is uniquely predictable no matter what phenotypes a given population may have started with in the distant past-a much stronger version than the one evolutionary psychologists typically think themselves committed to.

Rellihan notes that the usual justification by evolutionary psychologists for the use of adaptive thinking is given by appealing to a rather modest form of adaptationism, to the effect that "the mind's adaptive complexity reveals it to be a product of selection" (Rellihan 2012, p. 245). But he argues that this justification is insufficient, for the mind's being an adaptation is only a necessary and not a sufficient condition for the validity of adaptive thinking. Even granted that most of our mind's features are designed to perform fine-tuned adaptive functions, it does not warrant the deducibility of those functions from hypothesized initial conditions. Therefore, much stronger assumptions are needed in order to be able to predict the psychological mechanisms possessed by modern humans on the basis of knowledge about the selection pressures faced by our ancestors. ${ }^{6}$ Then what are those assumptions?

First, Rellihan defines adaptive thinking succinctly as an inference strategy in accordance with the following formula:

From the fact that there was a significant selection pressure for organism $O$ to evolve trait $T$, infer that $O$ has evolved T. (Rellihan 2012, p. 249) ${ }^{7}$

Then he introduces the notion of an "adaptive landscape" as a graphical way to represent what this inference strategy will amount to. Imagine an $N$-dimensional graph with a separate axis for each conceivable phenotypic property. Movement along an axis corresponds to quantitative change of the value of its associated property. Thus, such phenotypic properties as height, beak size, linguistic capacity, speaking in general terms, are represented by corresponding axes. By adding one extra axis representing the relative fitness of the organism that comprises those properties, we will then get an adaptive landscape for the species concerned.

In this landscape, organisms are represented as points on the surface, populations as clusters of associated points,

\footnotetext{
${ }^{6}$ In this sense, the issue concerning the accuracy with which to identify those ancient problems is not as fundamental to Rellihan as this issue of predictability (deducibility) of solutions via adaptive thinking. Even if Machery's idea of a bootstrap strategy makes it plausible that backward-looking reverse engineering can assist forward-looking adaptive thinking in better identifying the initial conditions of human evolution, it will not affect his point here that "even if we can identify these initial conditions, very little can be inferred about our evolved psychology" (Rellihan 2012, p. 273).

7 In contrast, reverse engineering is defined by the following formula: "From the fact that trait $T$ is well designed for $\Phi$-ing, infer that $T$ is an adaptation for $\Phi$-ing" (Rellihan 2012, p. 248).
} 
evolution as the process in which these clusters travel across the surface, and evolution by natural selection as the process in which populations ascend fitness peaks. Nonadaptive evolutionary change such as through genetic drift is represented as wandering about along a contour line. And saltatory evolution, say by means of macromutations, if any, is represented as a leap to a different position far from the current one. Thus, the power of selection can be thought of as the extent to which a population's evolutionary trajectory is determined by the surrounding topography as a gradual hill-climbing process without leaps. Since adaptationism is a position that sees the power of selection as by far the most predominant of all the factors influencing evolution, adaptationists insist the trajectory be mostly (if not exclusively) determined by the topography (Orzack and Sober 1994).

Now evolutionary psychology is committed to adaptive thinking, a special type of adaptationism with a predictive focus, according to which a population's evolutionary trajectory, and hence its destination all the way from its current position, can be predicted mostly by taking the power of selection into account. Therefore, according to Rellihan, in order to justify the use of adaptive thinking, we must presuppose the validity of what he calls "strong adaptationism," defined as follows:

The evolutionary path of a population across the adaptive landscape is largely determined by (and therefore predictable on the basis of) the population's current position on the landscape together with the neighboring topography of the landscape. (Rellihan 2012, p. 256)

However, when we begin to take into account the actual constraints of the epistatic interaction between component phenotypes-what Kauffman (1995) calls "conflicting constraints"- the fitness contribution of one trait becomes contingent upon the presence or absence of another one, thus, contributions by different traits become more and more nonadditive. Accordingly, the landscape becomes increasingly rugged with many a local optimum appearing here and there.

In such a situation, it will be difficult to predict the evolutionary destination (and the trajectory leading to it) solely on the basis of the landscape's topography plus the current position of the population. If the landscape were simple and smooth, such that there were only one global peak as with Mt. Fuji, we would not have to specify the point of departure and the intermediary pathway in order to predict that a population would eventually arrive at the peak; from anywhere on the landscape there could always be found a continuously uphill route leading to the peak. In contrast, if the landscape gets more and more rugged as a result of epistasis, it gets increasingly harder to predict to which peak a population will eventually ascend and along what route.
What does this all amount to for evolutionary psychology? If the actual landscape involved in the evolutionary history of the human mind happens to be simple and smooth, with a single optimal solution specifiable to the ancient problems, no matter what psychological phenotypes our ancestral population was initially possessed of, it is assured of evolving that solution over time, as the orthodoxy of evolutionary psychology teaches us. On the other hand, if the landscape becomes more or less rugged, just being able to specify the initial problems is not nearly sufficient to predict the end products unless at the same time sufficient information is provided both about the state of ancestral phenotypes and the sequential intermediary stages of their evolution.

What then does the actual landscape look like? Rellihan argues that there is evidence that it has always been considerably rugged. In the original "NK model" put forward by Kauffman and Levin (1987), where $N$ represents the number of distinct components of the system-genes in the case of a genotype, traits in the case of a phenotype-and $K$ the degree of epistatic interaction between them, $K=0$ corresponds to the case where the landscape is smooth, containing a single global peak, whereas at $K=2$ "the landscape already begins to resemble the French Alps," and at the extreme of $K=N-1$ "it looks more like a bed of nails." The number of peaks increases exponentially as either $K$ or $N$ increases. According to Kauffman and Levin's mathematical model, there will be $10^{28}$ peaks when $N=100$ and $K=99$, and $10^{48}$ peaks when $N=1024$ and $K=1$. In contrast, the human genome contains around 25,000 to 30,000 genes and our phenotypes consists of thousands of distinguishable traits (Rellihan 2012, p. 260).

The lesson to be drawn from the consideration above is that although the use of adaptive thinking is essential in evolutionary psychology theorizing, the condition in which it can be justified is extremely limited.

Recall Wimsatt's argument that using heuristics is applying a kind of reductionistic research strategy for reducing the complexity of the system by introducing simplifications or idealizations. This disregard of the effects of epistasis constitutes one of the core simplifying assumptions set in the discipline by its pioneers and henceforth having been inherited by inertia, as it were, by their followers. This is not an innocuous but a pernicious type of simplification, for it misleads us into accepting a caricatured picture of evolution on the grounds of the irresistibility of a naive intuition that the mind's adaptive complexity reveals it to be a product of selection.

Adaptive thinking is an inference justifiable only in idealized conditions: the extent to which epistatic interaction occurs should be extremely low, as we just saw above. Besides that, evolutionary forces other than selection should be negligible, ancient selection pressures should have remained robust at least until the relevant psychological 
adaptations were set in place, and there should not be any major developmental constraints that compromise the optimizing force of natural selection. Accordingly, if adaptive thinking is to serve as an effective heuristic that can significantly reduce the hypothesis space by picking out promising candidates worthy of serious consideration, those idealized conditions must have approximated the historical conditions in which evolution of the human mind has actually taken place. On the other hand, if these conditions are too ideal for any actual historical condition to come close to, adaptive thinking will not be serviceable even as an effective heuristic.

This state of affairs may be better understood with the help of the following analogous situation. Galileo's law of free fall obtains only in idealized conditions where there are no other forces than gravity that act on the object. The reason that this law can approximate the behavior of an actual object falling in the air is because the effect of air resistance is negligible compared to the force of gravity. However, the stronger the viscosity of the surrounding medium becomes, the less reliable will the application of this idealization to an actual condition be, such that the law can no longer predict the movement of an object sinking in the water, for instance.

\section{The Relationship Between Adaptive Thinking and Heuristics}

Now we will get back to the initial issue of whether evolutionary psychology can be construed as a heuristic program. What has become of the claim that since empirical data gets the last word in confirming hypotheses, adaptive thinking can settle for the minor status of just a heuristic device?

First, we want to ensure that adaptive thinking constitutes a core heuristic in evolutionary psychology, which even its proponents would willingly endorse.

For instance, Machery argues that tracing back to the historical origins of the trait by means of adaptationist thinking — whether it be in a forward- or backward-looking manner-is what provides evolutionary psychology with its "originality" or raison d'être:

So far, there is no difference between evolutionary psychologists' hypotheses and the hypotheses developed by other psychologists. What distinguishes the structure of evolutionary psychologists' theories is a third, distinctive level of hypothesis: Evolutionary psychologists attempt to identify the origins of the psychological traits under consideration. (Machery forthcoming, p. 15; emphasis in original)

That is, without an adaptationist perspective, evolutionary psychology would not deserve the name of evolutionary psychology, for then it would be deprived of the critical tool to identify the historical origins.

Of course, heuristics in evolutionary psychology do not have to be confined to adaptive thinking. A variety of sources of information can serve as heuristics so long as they can generate some testable hypotheses. Machery mentions the usefulness of nonselectionist sources of information coming from such areas as cross-species comparisons, hunter-gatherer studies, and paleoanthropology. Nonetheless, he treats them as "constraints" that only play supplementary roles to curb the speculative character of adaptive thinking. ${ }^{8}$ This suggests that unless adaptive thinking constitutes an integral part that binds up all these auxiliary sources, evolutionary psychology may end up with a mere hodgepodge of heterogeneous bodies of knowledge, such that its disciplinary integration will be jeopardized.

Second, as we noted time and again, one of the important functions heuristics are expected to perform is to narrow down the hypothesis space by sorting out a handful of promising hypotheses more likely to be true from the rest of the worthless ones. But then it follows that even heuristics already have to have some justificatory function-not just a discovering one. Therefore, if adaptive thinking functions as a core heuristic in evolutionary psychology, as we noted above, it cannot just settle for an innocuous role as generator of whatever hypotheses make evolutionary sense; rather, it has to take on a more active role in turning how-possibly explanations to how-actually ones as much as possible in advance of final testing. This is a reassurance of our previous point that the probability of hypotheses becoming true is a function of both the reliability of the context of discovery and the conclusiveness of that of justification and hence that we cannot draw a sharp line between the two. ${ }^{9}$

\footnotetext{
${ }^{8} \mathrm{He}$ also includes "middle-range evolutionary theories" as one of the constraints to a forward-looking heuristic. I will leave it out here, however, for they seem not so much constraints to adaptive thinking as more basic evolutionary theories themselves. For instance, Trivers's theory of parental investment (Trivers 1972), which Machery takes as an exemplar of middle-range evolutionary theories that constrain forward-looking heuristics such as Buss's theory of the human mating strategy, seems to actually function as a major premise to deduce Buss's theory combined with a minor premise of specifically human cases, rather than as a constraint imposed on it from without.

${ }^{9}$ Rellihan argues in this context, "One and the same inference procedure [i.e., adaptive thinking as a theory-driven inference strategy] would be considered reliable if it produced true beliefs with an eighty percent frequency and merely an effective heuristic if it produced true beliefs with, say, a twenty percent frequency. Heuristics are simply less reliable inference strategies; inference strategies are simply more reliable heuristics" (Rellihan 2012, p. 253; clarification added). This is another way of stating that adopting "Oh, it is just a heuristic!" tactics cannot be an excuse for having yet to provide sufficient grounds for accepting a hypothesis.
} 
Since Hans Reichenbach (1938) proposed it, the notion of the "context distinction" between those of discovery and justification had been predominant in the mainstream philosophy of science throughout the 20th century (Schickore 2018). In actual practice in science, however, the distinction cannot necessarily be drawn that neatly; this distinction has rather been utilized for sanctifying the role of philosophy of science à la logical positivism than for describing real scientific practices.

For example, getting back to Goldfinch's formula of the adaptationist version of the hypothetico-deductive methodthat "if trait $T$ is an adaptation for $X$, trait $T$ should have configuration $C$, and so we should find phenomenon $P "$ - it can be schematically represented as follows:

$X \rightarrow T \rightarrow C \rightarrow P$.

Leaving off the intermediary stage $C$ and dividing the whole into two qualitatively distinct stages of the generation and confirmation of hypotheses, it can be represented as:

$\mathbf{X} \rightarrow \mathbf{T} \rightarrow \mathbf{P}$,

where the first part $X \rightarrow T$ may be called the context of discovery and the second part $T \rightarrow P$ the context of justification.

Here it might be argued that what happens in the context of justification screens off the information about what had happened in the context of discovery. ${ }^{10}$ That is, no matter in what way $T$ had been derived from $X$, once $T$ is proposed at all, all the relevant information for designing and conducting confirmatory research of $T$ should be sought in the semantic content of $T$ alone, thereby rendering the information about how $T$ is generated in the first place irrelevant. ${ }^{11}$

This seems to be what Goldfinch actually has in mind. For, in his argumentation, the part of the predictive project $X \rightarrow T$ is supposed to be carried on almost automatically: once an adaptive problem $(X)$ is given, somehow the necessary solutions to it $(T)$ are almost bound to be forthcoming. The explanatory gap between $X$ and $T$ is too easily bridged. Compared to all the weight he places on and pages he allocates to describing how the confirmation of the hypotheses heuristically generated by evolutionary psychology should be carried out reliably in relevant adjacent fields (Goldfinch 2015, Chap. 4), his lack of interest in this phase of how the

\footnotetext{
${ }^{10}$ For the idea of "screening-off" refer to Brandon (1982); Salmon (1971)

11 A typical example is the well-known case of discovery of the benzene ring by August Kekule: although it is reported that Kekule hit upon the idea of the benzene ring from a dream he had during his slumber, that episode is irrelevant to the scientific legitimacy of the idea so long as it is confirmed by a rigorous testing procedure.
}

solutions to given problems should be predicted reliably is noteworthy.

But proposing some trait as a candidate adaptation is not an easy task. As George Williams argued, "adaptation is a special and onerous concept that should be used only where it is really necessary" (Williams 1966, p. 4); that is, it should not be invoked when less onerous and more parsimonious explanations are sufficient to do the trick. Therefore, this way of tipping the scale of the weight of establishing scientific facts exclusively to the side of final testing, thereby downplaying the weight to be carried by reliable hypothesis generation, is unbalanced.

Goldfinch's underappreciation of the necessity of providing reliable evidential particulars in generating adaptationist hypotheses is understandable, considering that one of his primary targets is Robert Richardson's Evolutionary Psychology as Maladapted Psychology (2007), which presented an exactly opposite case to his position, and therefore it appears that Goldfinch could not take a sympathetic stance toward what Richardson had emphasized. In that book, Richardson dismisses evolutionary psychology as a collection of unfounded speculations, because evolutionary psychologists seldom provide historical details only with which their adaptationist hypotheses can be substantiated. He draws on Brandon's (1990) analysis on the evidential criteria that any "adaptation explanations" have to meet to qualify as reliable ones. They consist in providing historically informed evidential details concerning the following five conditions: (1) selection, (2) ecological factors, (3) heritability, (4) population structure, and (5) trait polarity. Without the information about, at least, several of these conditions, any adaptation explanations will remain as unreliable stories (Brandon 1990, Chap. 5; Richardson 2007, pp. 99f.). ${ }^{12}$

The same case for the necessity of basing hypotheses on reliable historical underpinnings could be made by the following consideration. One of the rivals of evolutionary psychology in its budding stage in the 1980s through the 1990s was contemporary cognitive psychology (on top of sociobiology, as I argued in the second section). The pioneers of evolutionary psychology of the time had to demonstrate their methodological superiority to cognitive psychologists by claiming that only an evolutionary perspective could provide deeper insights into the historical origin of the now apparently synchronic constitution of the human mind. This was supposed to be possible by having access to the

\footnotetext{
12 Griffiths's 1996 quite lucid piece titled "The Historical Turn in the Study of Adaptation" is also written throughout with the same spirit of stressing the need to heavily incorporate historical information into the study of adaptation, such as from the comparative method or cladistics, to give substance and credibility to adaptationist storytelling including evolutionary psychological ones (Griffiths 1996).
} 
vantage point of the ancient selection pressures imposed on our ancestors.

In those days, the brain structure remained a "black box" with the neural circuits inside almost invisible. Although cognitive psychology had developed powerful techniques that provided clues to understanding it at levels above individual neurons, it still counted on quite indirect ways of investigation, such as stimulating the brain with images, sounds, or questions and inferring its structure from the corresponding outputs such as buttons pressed or boxes checked (Hagen 2002). Under such a situation, an evolutionary perspective could be a promising alternative for better approaching the structure and functions of the brain. The currently synchronic functional organization of the brain may be a close reflection of the survival and reproductive necessities in our ancestral environments. Studying the past remained much easier than studying brain wiring. It should be an evolutionary perspective "that sets the agenda for cognitive science, telling it what to look for and how to interpret what it finds" (Griffiths 2011, p. 405). That is, conjecturing past adaptive problems and hypothesizing solutions to them could provide heuristically useful targets for later more rigorous empirical research to zero in on in a search space otherwise too vast to search exhaustively. Thus, "The major insight of evolutionary psychology is that if you want to understand the brain, look deeply at the environment of our ancestors as focused through the lens of reproduction" (Hagen 2002, p. 520).

For example, in an effort to explain the so-called content effect on the Wason selection task, Cosmides and Tooby attempted to establish the methodological advantage of their "Social Contract Theory" over contemporary rival theories such as the "Pragmatic Reasoning Schemas" put forward by cognitive psychologists Patricia Cheng and Keith Holyoak (Cheng and Holyoak 1985). In doing so, they relied heavily on an evolutionary perspective for eliminating their rival theories; in one context, Cosmides argues that her social contract theory is based on the idea of domain-specific mechanisms while the rival theory is based on domain-general ones, and that evolutionary theory adjudicates in favor of the former:

The more important the adaptive problem, the more intensely selection should have specialized and improved the performance of the mechanism for solving it $[. .$.$] . Thus, the realization that the human$ mind evolved to accomplish adaptive ends indicates that natural selection would have produced specialpurpose, domain-specific mental algorithms including rules of inference for solving important and recurrent adaptive problems (such as learning a language [...]). (Cosmides 1989, p. 193)
I will not delve here into whether or not this way of appealing to evolutionary theory was legitimate, an issue that has already been given exhaustive consideration in the literature. ${ }^{13}$ What I want to stress here instead is that this way of discriminating its proprietary methodology from that of its rivals by appealing to an evolutionary (adaptationist) perspective was built in by its pioneers as one of the core identities of evolutionary psychology without which the discipline would not deserve the title. At the same time, however, this is only possible when adaptive thinking is substantiated with as sufficient historical underpinnings as possible.

\section{Conclusion}

Evolutionary psychology is, prima facie, going along the right track as a steady scientific discipline. A variety of psychological and behavioral traits of humans have been given evolutionary interpretations. Further, its methodology is extending beyond psychology into such surrounding areas as mental health, study of religion, criminology, consumer psychology, and so forth (cf. Buss 2016). For instance, the reinterpretations of mental disorders (on top of other diseases) in evolutionary medicine may be promising in that they can provide ultimate, etiological explanations for "why we get sick" (Nesse and Williams 1994), distinguished from, say, the typological classifications by methods of traditional psychiatry such as given in the DSM (Diagnostic and Statistical Manual of Mental Disorders). I am one of those who have a positive expectation that evolutionary psychology could eventually provide deeper understanding of our psychology and behaviors by bringing ultimate, evolutionary inquiries to bear on the study of proximate, mechanical causes.

Nevertheless, it is also true that pop hypotheses that attract media coverage have been constantly generated in some circles and disseminated without being put through rigorous tests. More importantly, even those hypotheses allegedly having been put through scientific confirmation are oftentimes more of a sort of reassurance of findings that are supposed to supervene on (or just correlate with) the hypothesized entities, rather than the confirmation of those entities themselves (like in the confirmation of jealousy modules

\footnotetext{
${ }^{13}$ Having said that, Elisabeth Lloyd's critical analysis of the argumentation of Cosmides and Tooby in this context is noteworthy. Lloyd argues that, although Cosmides and Tooby try to establish Cosmides's experiments designed to demonstrate the reality of the cheater detection module as crucial experiments that managed to decisively eliminate rival hypotheses (Cosmides 1989; Cosmides and Tooby 1994; Tooby and Cosmides 1989), "the ostensible links to evolutionary biology — rather than the experimental evidence—are doing much of the work of eliminating rival psychological hypotheses. Once the exaggerated and ill-reasoned claims are removed, the experiments appear to support a non-evolutionary psychological theory at least as strongly" (Lloyd 1999, p. 213).
} 
in Buss et al. 1992). Otherwise, the alleged confirmations are often artifacts resulting from using theoretical models as what Wimsatt calls "pattern-matching templates": in an attempt to test a theoretical model, more often than not the researcher tends to use it as a pattern to organize phenomena by classifying results according to whether or not they fit the model, thereby choosing the parameters to be measured not independently of the model (Wimsatt 2007).

Then, can this state of affairs be attributed to the issue of the research ethics or morality of some researchers who lack sufficient methodological awareness? Not necessarily. My view tends to be rather that some kind of vulnerability or instability is inherent in the methodology of evolutionary psychology itself that makes it prone to those kinds of errors. That is, it appears that practitioners in evolutionary psychology today are still being largely constrained by theoretical presuppositions that the pioneers of the discipline had to incorporate, rather hastily, in need of confronting their rivals in traditional psychology of the time by demonstrating the superiority of their methodology.

Since the end of the 20th, however, situations surrounding evolutionary biology from which evolutionary psychology heavily draws as its theoretical authority have changed drastically. The initial biasing assumptions inherent in the Modern Synthesis itself have been brought to the fore, such that its received view of evolution cannot be taken at face value today.

For instance, the theory of niche construction, or cultural evolution in general, teaches us that we humans can reconstruct our social, cultural, or even ecological environments in such a way that the altered environments can in turn exert feedback effects on the selection pressures relevant to our evolution, especially of our cognitive capacities (OdlingSmee et al. 2003). This can happen in such a relatively short time in evolutionary terms that Mother Nature has to adopt a tinkering expedient to exapt (or co-opt) preexisting structures for meeting the novel and urgent needs rather than creating adaptations from scratch (Gould and Vrba 1982). This makes the relevance of the Pleistocene EEA to the evolution of the human mind less significant than postulated by evolutionary psychologists.

In addition, research in epigenetics brought out that DNA modifications triggered by environmental changes organisms encounter pre- or postnatally play important roles in the developmental plasticity of various morphological and behavioral traits of animals, including human brain structures, and that some of these effects can be transmitted across generations without underlying changes in the DNA sequence (Jablonka and Lamb 1995, 2005; Meaney 2001; McGowan et al. 2009).

Adding further to the list of new trends of research that are diametrically opposed to the nativist leaning of evolutionary psychology, the discovery of neuroplasticity in neuroscience revealed that, rather than comprising full-blown domain-specific cognitive modules, the human brain houses rudimentary module-like neuronal assemblies that become the substrate for developmental processes to mold into individually idiosyncratic neuronal pattern by the dynamic reassembling mediated through learning or experiences (Merzenich and Jenkins 1995; Panksepp and Panksepp 2000).

Therefore, there is less and less need for present-day evolutionary psychologists to continue to be constrained by the historical limitations that the pioneers of the discipline had to settle for in order to weather the initial predicaments they faced by superficially assimilating the orthodoxies of Modern Synthesis at the time, before such new trends of life and behavioral sciences as touched on above began to truly have bearing on the study of human cognition and emotions. Nevertheless, many of today's pragmatically minded evolutionary psychologists seem to be indifferent to these kind of basic issues while engaging in the so-called puzzle-solving in the phase of normal science à la Kuhn.

With respect to the above-mentioned use of theoretical models as "pattern-matching templates," trying to fit the data to the models rather than the other way around, Wimsatt (2007, pp. 88-89) further states as follows:

these kinds of promotion of a theoretical or experimental model to a paradigm ... can defer for a long time the noticing or analyzing of questions that were far more obvious at the start of this line of investigation. This phenomenon - the increasing entrenchment of a theoretical or experimental paradigm - in part serves to explain why disciples of an approach are often far less flexible and far less methodologically conscious than the originators of that approach.

This statement is not explicitly addressed to evolutionary psychology, but the extent to which it is applicable to it is remarkable. Unless evolutionary psychologists become more aware of these issues and embark on pursuing more realityoriented - rather than doctrine-oriented - ways of establishing the science of the human mind, it may someday end up being remembered in history as one of the exemplary cases of degenerative research programs in the Lakatosian sense, comparable to phrenology. For a research program's being carried out in line with the typical formula of hypothesis-driven scientific reasoning is just a necessary—but not a sufficient—reason for it to qualify as a science in a productive and progressive state.

Acknowledgments This work was supported by a Grant-in-Aid for Scientific Research (C) from the Ministry of Education, Culture, Sports, Science and Technology of Japan (Grant Number: JP19K00277). I would like to thank the Numerical Algorithms Group and Editage for language and editing services.

Open Access This article is licensed under a Creative Commons Attribution 4.0 International License, which permits use, sharing, 
adaptation, distribution and reproduction in any medium or format, as long as you give appropriate credit to the original author(s) and the source, provide a link to the Creative Commons licence, and indicate if changes were made. The images or other third party material in this article are included in the article's Creative Commons licence, unless indicated otherwise in a credit line to the material. If material is not included in the article's Creative Commons licence and your intended use is not permitted by statutory regulation or exceeds the permitted use, you will need to obtain permission directly from the copyright holder. To view a copy of this licence, visit http://creativecommons .org/licenses/by/4.0/.

\section{References}

Andrews PW, Gangestad SW, Matthews D (2002) Adaptationism: how to carry out an exaptationist program. Behav Brain Sci 25(4):489-504

Bateson P (1994) The dynamics of parent-offspring relationships in mammals. Trends Ecol Evol 9:399-403

Brandon RN (1982) The levels of selection. PSA: Proceedings of the biennial meeting of the Philosophy of Science Association. 1982:315-323

Brandon RN (1990) Adaptation and environment. Princeton University Press, Princeton

Buller DJ (2005) Adapting minds: evolutionary psychology and the persistent quest for human nature. MIT Press, Cambridge

Buss DM (2000) The dangerous passion: why jealousy is as necessary as love and sex. Free Press, New York

Buss DM (2008) Evolutionary psychology: The new science of the mind, 3rd edn. Pearson/Allyn and Bacon, Boston

Buss DM, Larsen RJ, Westen D, Semmelroth J (1992) Sex differences in jealousy: evolution, physiology, and psychology. Psychol Sci 3:251-256

Buss DM (ed) (2016) The handbook of evolutionary psychology, vol 2: integrations. 2nd edn. Wiley, Hoboken

Caporael LR (1989) Mechanisms matter: the difference between socioblology and evolutionary psychology. Behav Brain Sci 12:17-18

Cheng PW, Holyoak KJ (1985) Pragmatic reasoning schemas. Cogn Psychol 17:391-416

Cosmides L (1989) The logic of social exchange: has natural selection shaped how humans reason? Studies with the Wason selection task. Cognition 31:187-276

Cosmides L, Tooby J (1987) From evolution to behavior: evolutionary psychology as the missing link. In: Dupré J (ed) The latest on the best: essays on evolution and optimality. MIT Press, Cambridge, pp 276-306

Cosmides L, Tooby J (1994) Beyond intuition and instinct blindness: toward an evolutionarily rigorous cognitive science. Cognition 50:41-77

Cosmides L, Tooby J, Barkow JH (1992) Introduction: evolutionary psychology and conceptual integration. In: Barkow J, Cosmides L, Tooby J (eds) The adapted mind: evolutionary psychology and the generation of culture. Oxford University Press, Oxford, pp 3-15

Davies PS (1999) The conflict of evolutionary psychology. Where biology meets psychology: philosophical essays. MIT Press, Cambridge, pp 67-81

Fox CW, Westneat DF (2010) Adaptation. In: Westneat DF, Fox CW (eds) Evolutionary behavioral ecology. Oxford University Press, Oxford, pp 16-25

Gigerenzer G, Selten R (2001) Bounded rationality: the adaptive toolbox. MIT Press, Cambridge
Goldfinch A (2015) Rethinking evolutionary psychology. Palgrave Macmillan, London

Gould SJ, Vrba ES (1982) Exaptation: a missing term in the science of form. Paleobiology 8:4-15

Griffiths PE (1996) The historical turn in the study of adaptation. Br J Philos Sci 47:511-532

Griffiths PE (2011) Ethology, sciobiology, and evolutionary psychology. In: Sarkar S, Plutynski A (eds) A companion to the philosophy of biology. Wiley-Blackwell, Malden, pp 393-414

Hagen EH (2002) Special design's centuries of success. Behav Brain Sci 25:519-520

Hagen EH (2016) Evolutionary psychology and its critics. In: Buss D (ed) The handbook of evolutionary psychology, vol 1: foundation, 2nd edn. Wiley, Hoboken, pp 136-160

Jablonka E, Lamb M (1995) Epigenetic inheritance and evolution: the Lamarckian dimension. Oxford University Press, Oxford

Jablonka E, Lamb M (2005) Evolution in four dimensions: genetic, epigenetic, behavioral, and symbolic variation in the history of life. MIT Press, Cambridge

Kauffman SA (1995) At home in the universe: the search for laws of self-organization and complexity. Oxford University Press, New York

Kauffman SA, Levin S (1987) Towards a general theory of adaptive walks on rugged landscapes. J Theor Biol 128:11-45

Laland KN, Brown GR (2011) Sense and nonsense: evolutionary perspectives on human behaviour, 2nd edn. Oxford University Press, New York

Lloyd EA (1999) Evolutionary psychology: the burdens of proof. Biol Philos 14:211-233

Machery E (forthcoming) Discovery and confirmation in evolutionary psychology. In: Prinz JJ (ed) The Oxford handbook of philosophy of psychology. Oxford University Press, Oxford

McGowan PO, Sasaki A, D’Alessio AC, Dymov S, Labonté B, Szyf M, Turecki G, Meaney MJ (2009) Epigenetic regulation of the glucocorticoid receptor in human brain associates with childhood abuse. Nat Neurosci 12:342-348

Meaney MJ (2001) Maternal care, gene expression, and the transmission of individual differences in stress reactivity across generations. Annu Rev Neurosci 24:1161-1192

Merzenich MM, Jenkins WM (1995) Cortical plasticity, learning and learning dysfunction. In: Julesz B, Kovacs I (eds) Maturational windows and adult cortical plasticity. Addison-Wesley, New York, pp 247-272

Nesse RM, Williams GC (1994) Why we get sick: the new science of Darwinian medicine. Vintage, New York

Odling-Smee FJ, Laland KN, Feldman MW (2003) Niche construction: the neglected process in evolution. Princeton University Press, Princeton

Orzack SH, Sober E (1994) Optimality models and the test of adaptationism. Am Nat 143:361-380

Panksepp J, Panksepp JB (2000) The seven sins of evolutionary psychology. Evol Cogn 6:108-131

Reichenbach H (1938) Experience and prediction: an analysis of the foundations and the structure of knowledge. University of Chicago Press, Chicago

Rellihan M (2012) Adaptationism and adaptive thinking in evolutionary psychology. Philos Psychol 25:245-277

Richardson RC (2007) Evolutionary psychology as maladapted psychology. MIT Press, Cambridge

Richerson P, Boyd R (2005) Not by genes alone: how culture transformed human evolution. University of Chicago Press, Chicago

Rose MR, Lauder GV (1996) Adaptation. Academic Press, San Diego

Salmon WC (1971) Statistical explanation. In: Salmon WC, Jeffrey RC, Greeno JG (eds) Statistical explanation and statistical relevance. University of Pittsburgh Press, Pittsburgh, pp 29-88 
Schickore J (2018) Scientific discovery. The Stanford Encyclopedia of Philosophy (Summer 2018 Edition) https://plato.stanford.edu/ archives/sum2018/entries/scientific-discovery. Accessed 2 Sept 2019

Smith S (2019) Is evolutionary psychology possible? Biol Theory 15:39-49

Sterelny K (1995) The adapted mind. Biol Philos 10:365-380

Sterelny K, Griffiths PE (1999) Sex and death: an introduction to philosophy of biology. University of Chicago Press, Chicago

Symons D (1992) On the use and misuse of Darwinism in the study of human behavior. In: Barkow JH, Cosmides L, Tooby J (eds) The adapted mind: evolutionary psychology and the generation of culture. Oxford University Press, Oxford, pp 137-159

Tooby J, Cosmides L (1989) Evolutionary psychology and the generation of culture, part I: theoretical considerations. Ethol Sociobiol 10:29-49

Tooby J, Cosmides L (1990) The past explains the present: emotional adaptations and the structure of ancestral environments. Ethol Sociobiol 11:375-424

Tooby J, Cosmides L (1992) The psychological foundations of culture. In: Barkow JH, Cosmides L, Tooby J (eds) The adapted mind: evolutionary psychology and the generation of culture. Oxford University Press, Oxford, pp 19-136
Tooby J, Cosmides L (1998) Evolutionizing the cognitive sciences: a reply to Shapiro and Epstein. Mind Lang 13:195-204

Trivers R (1972) Parental investment and sexual selection. In: Campbell B (ed) Sexual selection and the descent of man. Aldine DeGruyter, Chicago, pp 136-179

Trivers R (1974) Parent-offspring conflict Am Zool 14:249-264

Tversky A, Kahneman D (1974) Judgment under uncertainty: heuristics and biases. Science 185:1124-1131

Williams GC (1966) Adaptation and natural selection: a critique of some current evolutionary thought. Princeton University Press, Princeton

Wimsatt WC (2007) Re-engineering philosophy for limited beings: piecewise approximations to reality. Harvard University Press, Cambridge

Publisher's Note Springer Nature remains neutral with regard to jurisdictional claims in published maps and institutional affiliations. 